\title{
Critical role of macrophages in the marginal zone in the suppression of immune responses to apoptotic cell-associated antigens
}

\author{
Yasunobu Miyake, ${ }^{1}$ Kenichi Asano, ${ }^{1,2}$ Hitomi Kaise, ${ }^{1}$ Miho Uemura, ${ }^{1}$ \\ Manabu Nakayama, ${ }^{3}$ and Masato Tanaka ${ }^{1}$ \\ 1Laboratory for Innate Cellular Immunity, RIKEN Research Center for Allergy and Immunology, Yokohama, Kanagawa, Japan. \\ 2Department of Nephrology, Graduate School of Medicine, Tokyo Medical and Dental University, Bunkyo, Tokyo, Japan. \\ ${ }^{3}$ Laboratory of Genome Technology, Department of Human Genome Technology, Kazusa DNA Research Institute, Kisarazu, Chiba, Japan.
}

\begin{abstract}
Injection of apoptotic cells can induce suppression of immune responses to cell-associated antigens. Here, we show that intravenous injection of apoptotic cells expressing a fragment of myelin oligodendrocyte glycoprotein (MOG) reduced MOG-specific $T$ cell response and prevented the development of EAE. Since injected apoptotic cells accumulated initially in the splenic marginal zone (MZ), the role of macrophages in the MZ in immune suppression was examined using transgenic mice in which these cells could be transiently deleted by diphtheria toxin (DT) injection. DT-treated mice became susceptible to EAE even though MOG-expressing apoptotic cells were preinjected. Deletion of the macrophages caused delayed clearance of injected dying cells in the MZ. In wild-type mice, injected apoptotic cells were selectively engulfed by $\operatorname{CD} 8 \alpha^{+} \mathrm{DCs}$, which are responsible for suppression of immune responses to cell-associated antigens. In contrast, deletion of macrophages in the MZ caused aberrant phagocytosis of injected dying cells by CD8 $\alpha^{-} C D 11 b^{+}$DCs. These results indicate that macrophages in the MZ regulate not only efficient clearance of apoptotic cells but also selective engulfment of dying cells by $\mathrm{CD} 8 \alpha^{+}$DCs and that functional failure of these unique macrophages impairs suppression of immune responses to cell-associated antigens.
\end{abstract}

\section{Introduction}

Apoptotic cell death is a critical and evolutionally conserved process for elimination of unnecessary cells $(1,2)$. After undergoing apoptosis, cell corpses are rapidly recognized and phagocytosed by professional phagocytic cells such as macrophages and DCs (3-5). Rapid removal of apoptotic cells by phagocytes prevents the release of potentially toxic or immunogenic materials from dying cells. In mammals, the recognition and engulfment of apoptotic cells is an intricate process, involving a number of molecules on phagocytes (6, 7). Participation of some of these molecules in apoptotic cell clearance in vivo has been defined by analysis of gene-targeted mice. For example, a mutant mouse with a truncated cytoplasmic tail of the Mer receptor tyrosine kinase showed delayed clearance of dexamethasone-induced apoptotic cells in the thymus (8). The milk fat globule-EGF-factor 8 (MFG-E8) is secreted by macrophages and binds phosphatidylserine (PS) on apoptotic cells, enhancing their clearance by phagocytes (9). MFG-E8 is expressed in the tingible body macrophages (TBM) located in the germinal centers of spleen and lymph nodes (LNs). In MFG-E8-deficient mice, TBM are associated with a large number of apoptotic cells; however, most of the corpses are not engulfed by the macrophages, illustrating the importance of MFG-E8 in apoptotic cell clearance in the germinal center (10). Both Mer

Nonstandard abbreviations used: CMFDA, 5-chloromethylfluorescein diacetate; DT, diphtheria toxin; DTR, DT receptor; HB-EGF; heparin-binding EGF-like growth factor; KLH, keyhole limpet hemocyanin; MAdCAM-1, mucosal addressin cell adhesion molecule-1; MFG-E8, milk fat globule-EGF-factor 8; MMM, marginal metallophilic macrophage(s); MOG, myelin oligodendrocyte glycoprotein; MOG-L, MOG long fragment; MOG-S, MOG short fragment; $\mathrm{MZ}$, marginal zone; MZM, MZ macrophage(s); PS, phosphatidylserine; TBM, tingible body macrophage(s). Conflict of interest: The authors have declared that no conflict of interest exists. Citation for this article: J. Clin. Invest. 117:2268-2278 (2007). doi:10.1172/JCI31990. and MFG-E8 mutant mice spontaneously develop autoantibodies $(10,11)$, indicating that the efficient removal of apoptotic cells by phagocytes plays a critical role in the maintenance of self tolerance.

Consistent with these observations, it has been reported that injection of cell-associated exogenous antigens can induce tolerance to these antigens in mice. Liu et al. found that injection of apoptotic cells loaded with OVA led to OVA-specific immune tolerance (12). Sun et al. reported that transfusion of apoptotic splenocytes from the donor strain prevented rejection of heart allografts (13). In both cases, it is presumed that the injected apoptotic cells were phagocytosed by APCs in the spleen and that the APCs then presented foreign peptides derived from the apoptotic cells to T cells. Presentation in the absence of appropriate costimulatory signals could lead to antigen-specific T cell deletion or anergy. However, the precise mechanisms for induction of $\mathrm{T}$ cell tolerance to cell-associated antigens is unknown.

EAE is characterized by neurological impairment, such as a progressive paralysis that first affects the tail and hind limbs. EAE is a model of human multiple sclerosis and is produced in experimental animals by injecting them with CNS tissue homogenates or purified components of the myelin sheath with adjuvants. The immunization results in the activation of myelin-specific, autoreactive Th1 cells, which induce inflammation and demyelination in the CNS. Therefore, induction of antigen-specific $\mathrm{T}$ cell tolerance could be of therapeutic benefit for both the onset and progression of EAE.

In this study, we show that intravenous injection of apoptotic cells expressing a fragment of myelin oligodendrocyte glycoprotein (MOG) induced MOG-specific tolerance and prevented the development of EAE induced by MOG immunization. Protection from EAE was lost when PS on the surface of injected apoptotic cells was masked. Since injected apoptotic cells initially accumu- 


\section{Figure 1}

Expression of HB-EGF-MOG fusion proteins in W3/MOG cells. (A) Schematic diagrams of the HB-EGF-MOG fusion protein constructs. The amino acids 32-97 (HB-EGF-MOG-S) or 1-120 (HB-EGFMOG-L) of MOG are attached at the C terminus of the signal (SIG), extracellular (EC), and transmembrane (TM) domains of HB-EGF. (B) Expression of HB-EGF-MOG/FLAG on the cell surface of transformants. W3 cells and their transformants expressing HB-EGF-MOG-S (W3/MOG-S) or HB-EGF-MOG-L (W3/MOG-L) were stained with Alexa Fluor 488-conjugated anti-HB-EGF antibody and analyzed by flow cytometry. (C) Immunodetection of HB-EGF-MOG proteins in the transformants. W3 cells (lanes 1, 4, and 7) and W3 cells expressing MOG-S (lanes 2, 5, and 8) or MOG-L (lanes 3, 6, and 9) were lysed and analyzed by Western blotting (WB) with anti-HB-EGF antibody (lanes 1-3). In order to confirm the expression of full-length HB-EGF-MOG/FLAG fusion proteins, cell lysates were subjected to immunoprecipitation with an anti-FLAG antibody. Immunoprecipitates were analyzed by Western blotting with anti-HB-EGF (lanes 4-6) or anti-MOG antibodies (lanes 7-9). (D) Induction of apoptosis in W3 cells and their transformants. W3 cells and their transformants were treated with (solid line) or without (dashed line) recombinant Fas ligand for 4 hours. Cells were then stained with PE-conjugated annexin V and analyzed by flow cytometry. More than $95 \%$ of W3 and W3/MOG cells underwent apoptosis by treatment with Fas ligand.

lated in the marginal zone $(\mathrm{MZ})$ of the spleen, we examined the role of macrophages in the $\mathrm{MZ}$ for tolerance induction. For this purpose, we established human diphtheria toxin receptor (DTR) transgenic mice, in which macrophages in the MZ could be specifically ablated by means of the toxin receptor-mediated conditional cell knockout (TRECK) system (14). Analysis of these mice revealed that macrophages in the $\mathrm{MZ}$ regulated both the clearance of circulating apoptotic cells and the selective engulfment of dying cells by CD $8 \alpha^{+}$DCs. In the absence of these macrophages, the induction of tolerance to cell-associated antigens was severely impaired.

\section{Results}

Intravenous injection of MOG-expressing dying cells inhibits EAE progression. Immunization with $\mathrm{MOG}_{35-55}$ peptide emulsified in CFA induces EAE in C57BL/6J mice. To determine whether the delivery of MOG epitopes via apoptotic cells could prevent EAE, we established transformants expressing MOG fragments. To anchor the MOG fragment to the plasma membrane of cells, cDNA encoding $\mathrm{MOG}_{32-97}$ (MOG-S) or $\mathrm{MOG}_{1-120}$ (MOG-L) was fused with the extracellular and transmembrane regions of human heparin-binding EGFlike growth factor (HB-EGF) cDNA (Figure 1A). The expression plasmids for the chimeric proteins were introduced into mouse W3 cells (WR19L cells expressing mouse Fas). Flow cytometry analysis revealed that the chimeric proteins were expressed on the cell surface of W3/MOG-S and W3/MOG-L cells (Figure 1B). When whole cell lysates were analyzed by Western blotting with anti-HB-EGF antibody, W3/MOG-S and W3/MOG-L cells showed major bands of 26 and $28 \mathrm{kDa}$ and of 33 and $35 \mathrm{kDa}$, respectively (Figure 1C). Immunoprecipitation with anti-FLAG antibody followed by Western blotting with anti-HB-EGF antibody or anti-MOG antibody resulted in bands of the same size as those in lanes 2 and 3 (Figure 1C), indicating that full-length chimeric proteins were expressed in W3/MOG-S and W3/MOG-L cells. When the W3 cells and their transformants were treated with Fas ligand, they underwent apoptosis, and more than $95 \%$ of cells were stained positive with annexin $\mathrm{V}$ after 4 hours (Figure 1D).
A
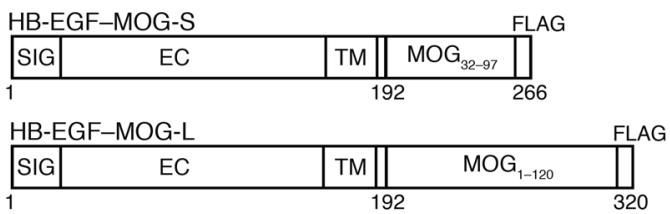

B

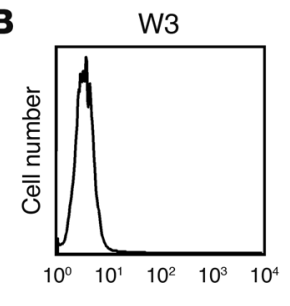

W3/MOG-S

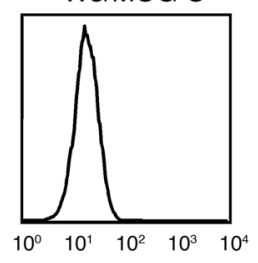

W3/MOG-L

C

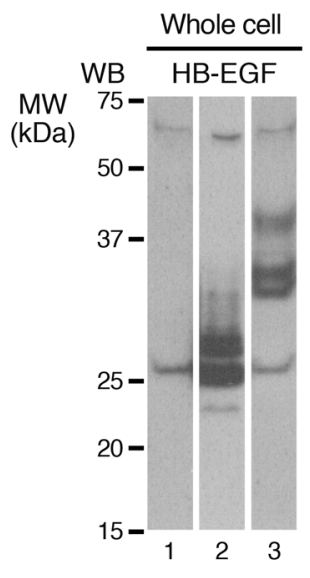

HB-EGF

D
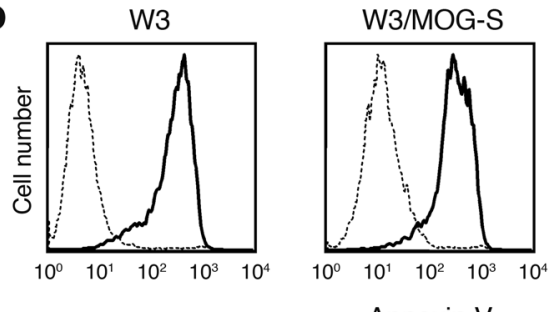

$\begin{array}{lll}7 & 8 & 9\end{array}$

Annexin V

We intravenously injected $2 \times 10^{7}$ of these apoptotic cells into C57BL/6J mice. Eight days later, the mice were immunized with $\mathrm{MOG}_{35-55}$ in CFA to induce EAE. As shown in Figure 2A, EAE was induced in nontreated mice with an average maximum clinical score of approximately 4 (see Methods). In striking contrast, prior injection of apoptotic W3/MOG-L or W3/MOG-S cells suppressed the development of EAE while injection of apoptotic unmodified W3 cells had no effect. Since W3/MOG-L cells showed a stronger effect on the inhibition of EAE than W3/MOG-S cells, W3/MOG-L cells were used in subsequent experiments.

We next examined the ability of different numbers of apoptotic W3/MOG-L cells to prevent EAE. With $2 \times 10^{7}$ or $1.2 \times 10^{7}$ cells, EAE was almost completely prevented while administration of $6 \times 10^{6}$ cells had a partial protective effect (Figure 2B), indicating that the effect of apoptotic W3/MOG-L cell injection on EAE was dose dependent.

We also determined whether administration of apoptotic W3/ MOG-L cells after $\mathrm{MOG}_{35-55}$ immunization had any effects on EAE 
A

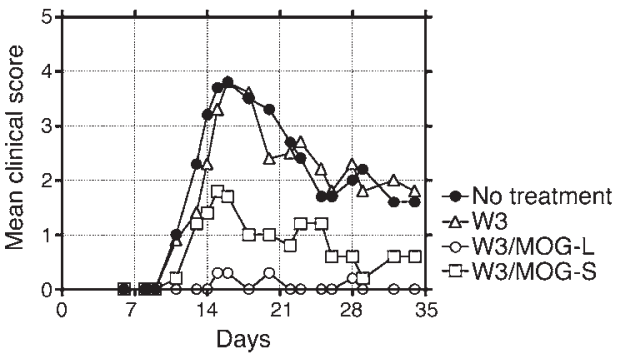

C

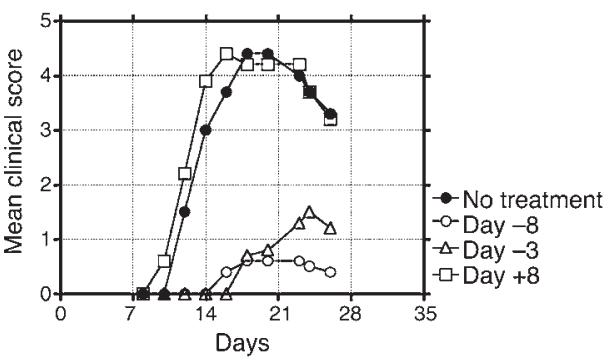

E

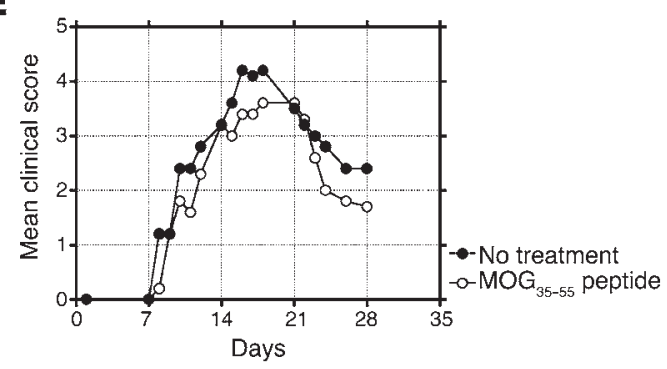

B

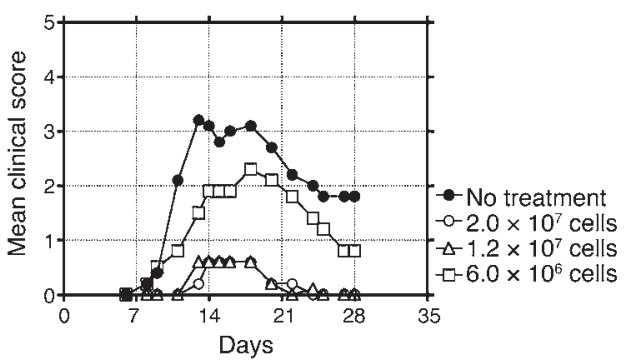

D

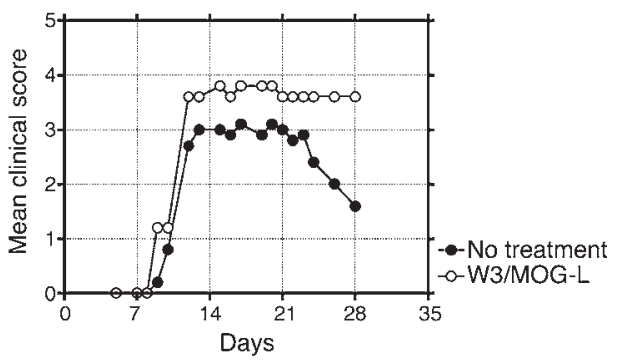

$\mathbf{F}$

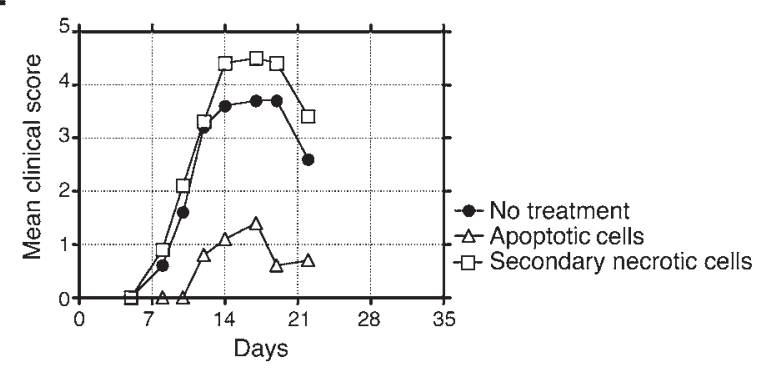

Figure 2

Intravenous administration of apoptotic W3/MOG cells prevents the progression of EAE. (A) We treated $2 \times 10^{7}$ W3, W3/MOG-S, and W3/MOG-L cells with Fas ligand to induce apoptosis. Then the treated cells were intravenously injected into mice 8 days prior to immunization. Mice were then immunized with $\mathrm{MOG}_{35-55}$ in CFA on day 0. (B) Dose-dependent effects of apoptotic cell injection on EAE. The indicated number of apoptotic W3/MOG-L cells was injected into mice on 4 days prior to immunization with $\mathrm{MOG}_{35-55}$ in CFA on day 0. (C) Pretreatment with apoptotic cells is required for suppression of EAE. Mice were treated with $2 \times 10^{7}$ apoptotic W3/MOG-L cells 8 or 3 days prior to immunization or 8 days afterwards. Immunization was on day 0. (D) Subcutaneous injection of apoptotic cells does not suppress EAE. We subcutaneously injected $2 \times 10^{7}$ apoptotic W3/MOG-L cells into the tail base 6 days before mice were immunized with $\mathrm{MOG}_{35-55}$ in CFA. (E) Intravenous injection of $\mathrm{MOG}_{35-55}$ peptide does not induce tolerance to $\mathrm{EAE}$. We intravenously injected $1 \mu \mathrm{g}$ of $\mathrm{MOG}_{35-55}$ peptide, which corresponds to more than 200 times the amount of MOG fragment expressed in $2 \times 10^{7}$ apoptotic W3/MOG-L cells in molar ratio, into mice. Four days later, the mice were immunized with $\mathrm{MOG}_{35-55}$ in CFA. (F) Secondary necrotic cells did not suppress EAE. W3/MOG-L cells were treated with Fas ligand either for 4 hours or 24 hours and were injected intravenously into mice 4 days prior to immunization with MOG $_{35-55}$ in CFA on day 0 . (A-F) The disease severity of each mouse was scored, and the mean clinical scores at the indicated times were plotted. These results are representative of 3 (A-D) or $2(\mathbf{E}$ and $\mathbf{F})$ independent experiments.

progression. As shown in Figure 2C, injection of apoptotic W3/ MOG-L cells either 8 or 3 days prior to immunization prevented EAE progression. On the other hand, when apoptotic W3/MOG-L cells were injected 8 days after $\mathrm{MOG}_{35-55}$ immunization, disease severity of EAE was not different from that in the mice without any pretreatment, indicating that pretreatment with the apoptotic W3/ MOG-L cells was required for the inhibition of EAE progression.

We next tested the effect of administration route of apoptotic W3/MOG-L cells. When $2 \times 10^{7}$ of the apoptotic W3/MOG-L cells were subcutaneously injected into the base of the tail before MOG immunization, there was no reduction in disease severity (Figure $2 \mathrm{D})$, indicating that intravenous injection was required for apoptotic W3/MOG-L cells to suppress EAE.

Since intravenous injection of a large amount of antigen or peptide can sometimes induce tolerance to the antigen $(15,16)$, we compared the tolerogenic effects of apoptotic W3/MOG-L cell injection with intravenous injection of $\mathrm{MOG}_{35-55}$ peptide. By Western blot analysis with the anti-FLAG antibody, we estimated that $2 \times 10^{7} \mathrm{~W} 3 / \mathrm{MOG}-\mathrm{L}$ cells contained approximately 2 pmol of the MOG fragment. Even with injection of 400 pmol of $\mathrm{MOG}_{35-55}$ peptide, 200 times more of the MOG fragment as a molar ratio, EAE progression was not inhibite $\mathrm{d}$ (Figure 2E), indicating that the uptake of the MOG fragment from apoptotic cells was required for efficient inhibition of EAE progression.

To reveal the characteristics required of dead cells to exert an immunosuppressive effect on cell-associated antigens, we examined the effects of secondary necrotic cell injection on EAE progression. W3/MOG-L cells treated with Fas ligand for 4 hours showed features characteristic of early-phase apoptosis (annexin Vpositive and PI-negative) and exhibited immunosuppressive 
A

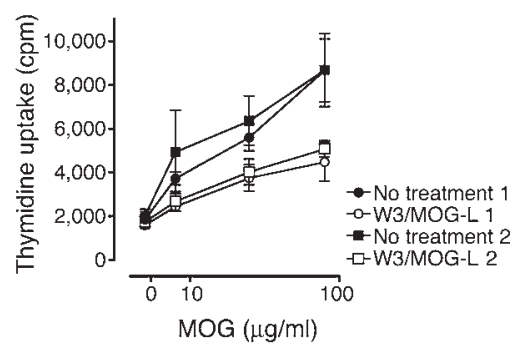

C

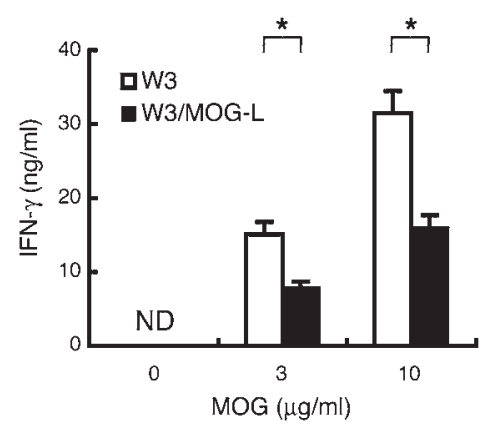

B

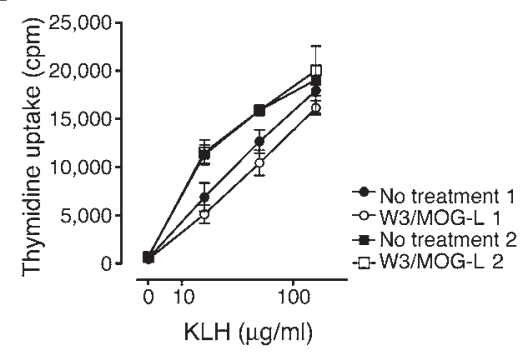

D

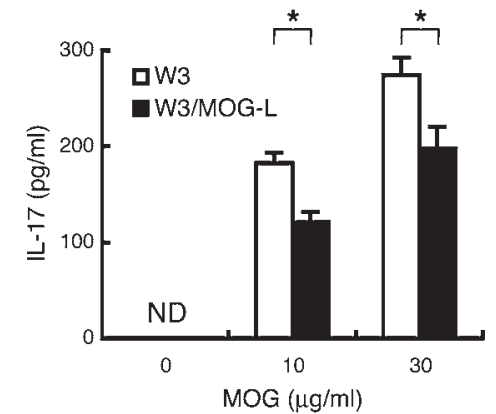

Figure 3

Administration of apoptotic W3/MOG cells induced antigen-specific $\mathbf{T}$ cell unresponsiveness. ( $\mathbf{A}$ and B) We intravenously injected $2 \times 10^{7}$ apoptotic W3/ MOG-L cells into mice. Three days later, $100 \mu \mathrm{g}$ of $\mathrm{MOG}_{35-55}(\mathbf{A})$ or $\mathrm{KLH}$ (B) in CFA was injected subcutaneously into the bilateral foot pads. Popliteal and inguinal LNs were collected from the mice 5 days after immunization. In vitro, $5 \times 10^{5} \mathrm{LN}$ cells were restimulated with $\mathrm{MOG}_{35-55}(\mathbf{A})$ or $\mathrm{KLH}(\mathbf{B})$ for 70 hours. $T$ cell proliferation was quantified based on the $\left[{ }^{3} \mathrm{H}\right]$ thymidine uptake in the last 20 hours of the culture. Mean values are shown with SD. Numbers indicate mouse 1 and mouse 2. (C and D) Apoptotic W3/MOG-L cell injection reduces IFN- $\gamma$ and IL-17 production in splenocytes. We intravenously injected $2 \times 10^{7}$ apoptotic W3 or W3/MOG-L cells into mice 4 days prior to immunization of $\mathrm{MOG}_{35-55}$ on day 0 . Splenocytes obtained 10 days after immunization were restimulated in vitro with $\mathrm{MOG}_{35-55}$ for 72 hours. Production of IFN- $\gamma$ (C) and IL-17 (D) was measured by ELISA. Mean values are shown with SEM. ${ }^{*} P<0.05$. These results are representative of 3 independent experiments. effects as described above. On the other hand, when W3/MOG-L cells were treated with Fas ligand for 24 hours, the cells became annexin $\mathrm{V}$-positive and PI-positive, indicating that they underwent secondary necrosis. As shown in Figure 2F, intravenous injection of secondary necrotic W3/MOG-L cells did not suppress EAE progression. These results clearly indicate that suppression of immune responses to cell-associated antigens requires exposure to early-phase apoptotic cells.

$M O G$-specific T cell responses in mice injected with apoptotic W3/MOG-L cells. We next determined whether intravenous injection of apoptotic W3/MOG-L cells reduced the activation of MOG-specific $\mathrm{T}$ cells. We intravenously injected $2 \times 10^{7}$ apoptotic W3/MOG-L cells into mice. Three days after injection, $\mathrm{MOG}_{35-55}$ in CFA was subcutaneously injected into the foot pads. Then the cells from the draining LNs were restimulated with $\mathrm{MOG}_{35-55}$ in vitro. As shown in Figure 3A, MOG-specific T cell response was greatly diminished in the mice pretreated with apoptotic W3/MOG-L cells. We also examined the effects of apoptotic cell injection on $\mathrm{T}$ cell responses to an irrelevant antigen. Apoptotic W3/MOG-L cells were intravenously injected into mice, and then keyhole limpet hemocyanin (KLH) in CFA was injected subcutaneously into the foot pads as above. As shown in Figure 3B, the KLH-specific $\mathrm{T}$ cell response was unaffected by pretreatment with apoptotic W3/ MOG-L cells. We also analyzed the effect of apoptotic cell injection on IFN- $\gamma$ and IL-17 production by splenocytes. Apoptotic W3 or W3/MOG-L cells were intravenously injected 4 days before immunization with $\mathrm{MOG}_{35-55}$ on day 0 . At day 10 , splenocytes were restimulated in vitro with $\mathrm{MOG}_{35-55}$. Pretreatment of W3/MOG-L cells significantly reduced IFN- $\gamma$ and IL-17 production by restimulated splenocytes (Figure 3, $\mathrm{C}$ and D). Taken together, these results indicate that injection of apoptotic W3/MOG-L cells reduces antigen-specific $\mathrm{T}$ cell responses to MOG.

PS-dependent phagocytosis of apoptotic W3/MOG-L cells is required for induction of tolerance. It seems likely that intravenously injected apoptotic W3/MOG-L cells are engulfed by APCs in the spleen and the APCs then present a MOG fragment for induction of tolerance. To engulf apoptotic cells, phagocytes must recognize ligands found on apoptotic but not on living cells. One such ligand is PS, which is exposed on the outer leaflet of the plasma membrane when cells undergo apoptosis. We have previously shown that an MFG-E8 protein with a point mutation in an RGD motif (D89E) inhibited apoptotic cell engulfment by masking PS and that intravenous injection of D89E induced the production of autoantibodies in mice by inhibiting apoptotic cell clearance (17). We next examined the effects of D89E on suppression of EAE by apoptotic cells. We injected $1 \times 10^{7}$ apoptotic W3/MOG-L cells into mice together with $10 \mu \mathrm{g} / \mathrm{ml}$ of D89E 3 days prior to immunization of $\mathrm{MOG}_{35-55}$ in CFA on day 0 . As shown in Table 1, the suppressive effects of apoptotic W3/MOG-L cells were largely, but
Table 1

Effect of MOG-expressing apoptotic cells on EAE

\begin{tabular}{lcccc}
\hline Mice & Treatment & & \\
C57BL/6J & Incidence & Day of onset & \\
& None & $15 / 15$ & $11.6 \pm 0.5$ & Mean maximal score $^{B}$ \\
& W3/MOG-LC & $14 / 15$ & $14.1 \pm 0.9$ & $4.1 \pm 0.3$ \\
& W3/MOG-LC+ D89E & $15 / 15$ & $12.8 \pm 0.9$ & $2.1 \pm 0.2^{\mathrm{D}}$ \\
CD11c-DTR & W3/MOG-LC + E1E2PT & $10 / 12$ & $11.5 \pm 0.7$ & $3.5 \pm 0.4^{\mathrm{E}}$ \\
& -DT None & $9 / 9$ & $10.0 \pm 0.5$ & $2.3 \pm 0.5^{\mathrm{F}}$ \\
& -DT W3/MOG-LH & $6 / 9$ & $14.2 \pm 1.3$ & $0.2 \pm 0.3^{\mathrm{G}}$ \\
& +DT' None & $14 / 15$ & $12.0 \pm 0.8$ & $3.2 \pm 0.6$ \\
& +DT' W3/MOG-LH & $15 / 16$ & $13.1 \pm 0.7$ & $3.0 \pm 0.5$
\end{tabular}

AMice were injected with apoptotic W3/MOG-L cells 4 days before immunization on day $0 .{ }^{B}$ Data are presented as mean \pm SEM. ${ }^{C} 1 \times 10^{7}$ cells. DNone versus W3/MOG-L, $P<0.005$. EW3/MOG-L versus W3/MOG-L + D89E, $P<0.005$. FNone versus W3/MOG-L + E1E2PT, $P<0.005$. G-DT none versus -DT W3/MOG-L, $P<0.005$. ${ }^{2} 2 \times 10^{7}$ cells. 'DT $5 \mu \mathrm{g} / \mathrm{kg}, 24$ hours. 
Figure 4

Generation of CD169-DTR mice. (A) Schematic diagram of the CD169-DTR targeting construct. Exons of the CD169 gene are indicated by solid boxes. Human DTR (hDTR) cDNA, the loxP-franked neomycin resistant gene (Neo), and the thymidine kinase gene (TK) are indicated by open boxes. The probe used for Southern blot analysis is indicated as a solid line together with the predicted hybridizing fragments. (B) Genomic Southern blot analysis of wild-type (+/+) and CD169-DTR (+/CD169DTR) mice using a $\mathrm{Bgl}$ II digest in combination with the indicated probe. (C) Depletion of macrophages in the MZ by DT administration in CD169-DTR mice. The indicated amount of DT was intraperitoneally injected into wild-type $(+/+)$ or CD169-DTR (+/CD169DTR) mice. Spleens were obtained from these mice 48 hours later. Spleen sections were immunostained with CD11b, ER-TR9, CD169, and CD11c for red pulp macrophages, MZM, MMM, and DCs, respectively. (D) We intraperitoneally injected $10 \mu \mathrm{g} / \mathrm{kg}$ of DT into wild-type mice $(+/+)$ and CD169-DTR (+/CD169DTR) mice. Spleens were obtained from these mice on the indicated days after DT administration and immunostained with ER-TR9 and CD169. Original magnification, $\times 100(\mathbf{C}$ and $\mathbf{D})$.
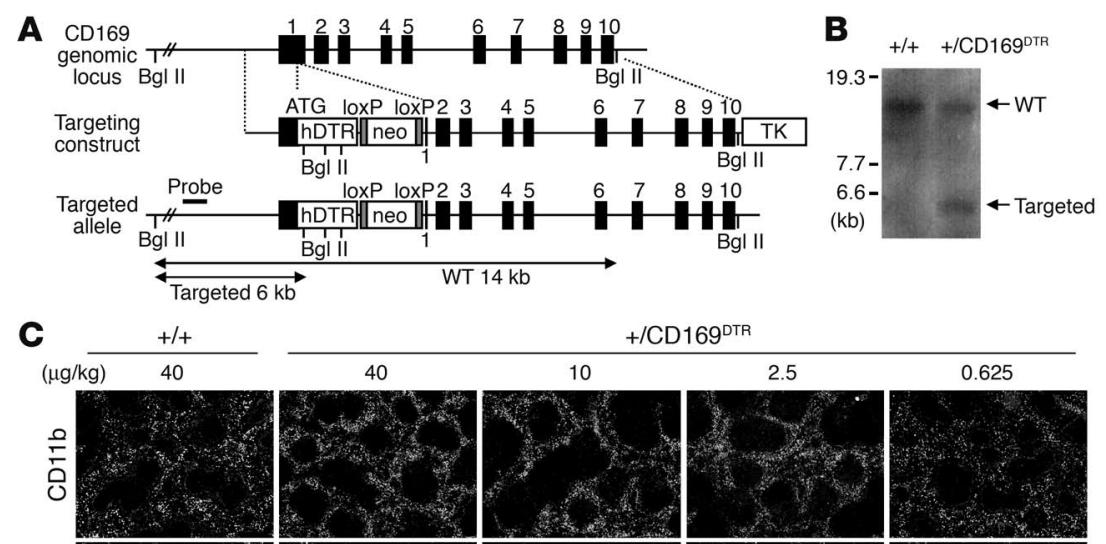

$169^{\text {DTR }}$
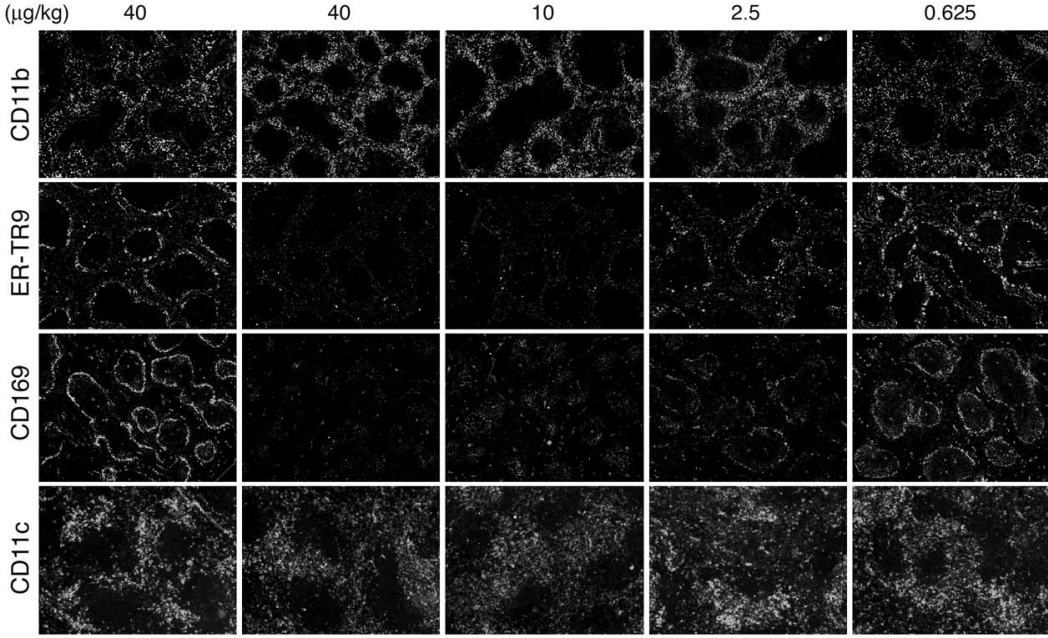

D
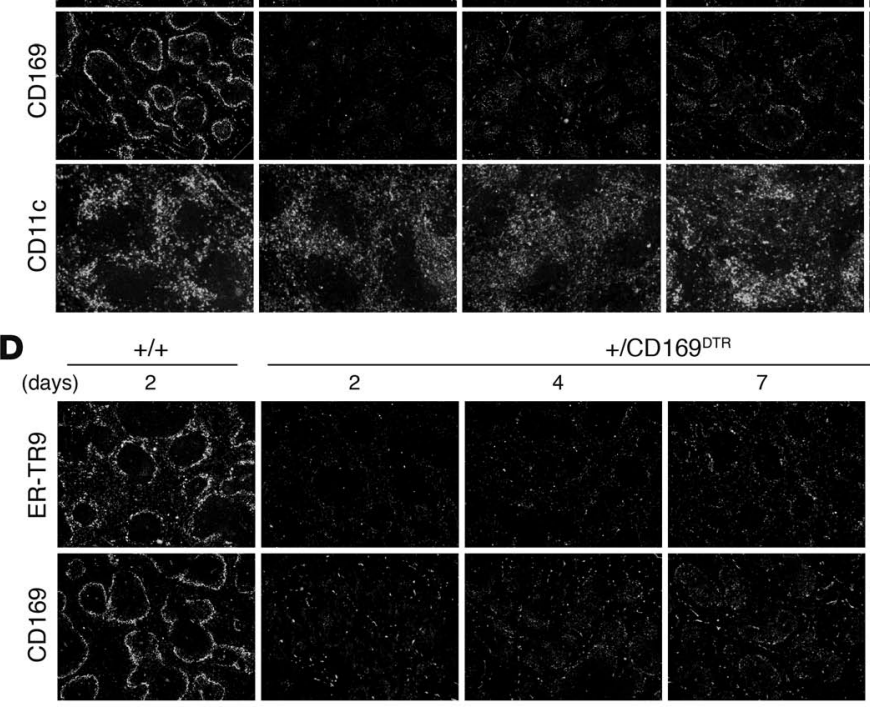

+/CD169
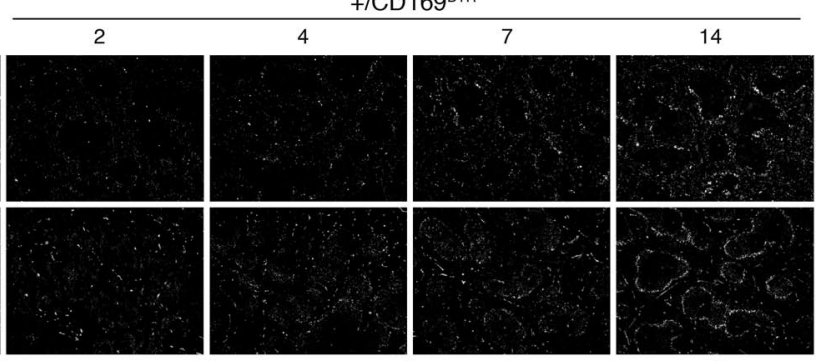

not completely, eliminated when the mice were injected with D89E while the suppressive effects were not affected by coinjection with E1E2PT, another MFG-E8 mutant protein that does not bind to PS. This result suggests that PS-dependent engulfment of apoptotic W3/MOG-L cells is required, at least in part, for the inhibitory effects on EAE induction.

EAE suppression is defective in CD11c-DTR mice. To determine which phagocytic cell type is required for apoptotic cell-mediated Agspecific tolerance, we first used CD11c-DTR mice. In these mice, a single administration of DT results in depletion of CD $11 \mathrm{c}^{+} \mathrm{DCs}$ (18). DT was intraperitoneally administrated into the mice 11 days before immunization. Twenty-four hours after the DT injection, by which time the CD11 $\mathrm{c}^{+} \mathrm{DCs}$ were depleted, $2 \times 10^{7}$ apoptotic W3/MOG-L cells were intravenously injected. On day 0, when DCs had completely recovered, mice were immunized with $\mathrm{MOG}_{35-55}$ in CFA. Injection of apoptotic W3/MOG-L cells did not suppress EAE in the DT-treated CD11c-DTR mice while DT treatment of wild-type mice had no effects on the induction of tolerance to MOG (Table 1 and data not shown). These results suggest that CD11 $\mathrm{c}^{+}$DCs are responsible for apoptotic cell-mediated Agspecific tolerance. However, Probst et al. recently reported that a single injection of DT into CD11c-DTR mice resulted not only in efficient and transient depletion of DCs but also in complete and protracted depletion of the MZ macrophages (MZM) and marginal metallophilic macrophages (MMM), both of which were normally located in the MZ of spleen (19). Therefore, we were not able to exclude the possibility that depletion of macrophages in the $\mathrm{MZ}$ affected the apoptotic cell-mediated Ag-specific tolerance in DT-treated CD11c-DTR mice.

Generation of CD169-DTR mice. In addition to the results with CD11c-DTR mice, we observed that injected apoptotic cells first accumulated in the $\mathrm{MZ}$ of spleen. These results prompted us to determine the role of macrophag es in the splenic MZ for induction of tolerance to apoptotic cell-associated antigens. For this purpose, we established transgenic mice in which these cells were transiently depleted by DT administration. As shown in Figure 4A, human DTR cDNA was introduced into the CD169 sialoadhesin gene, which is specifically expressed in $\operatorname{MMM}(20,21)$. Homologous recombination of CD169-DTR mice was confirmed by Southern blot analysis (Figure 4B).

We first examined what cell types in the spleen were depleted in CD169-DTR mice by DT injection. Since by immunohistochemistry the CD169 antigen is exclusively detected in the MMM, we expected a selective deletion of MMM in DT-treated CD169-DTR mice 
A

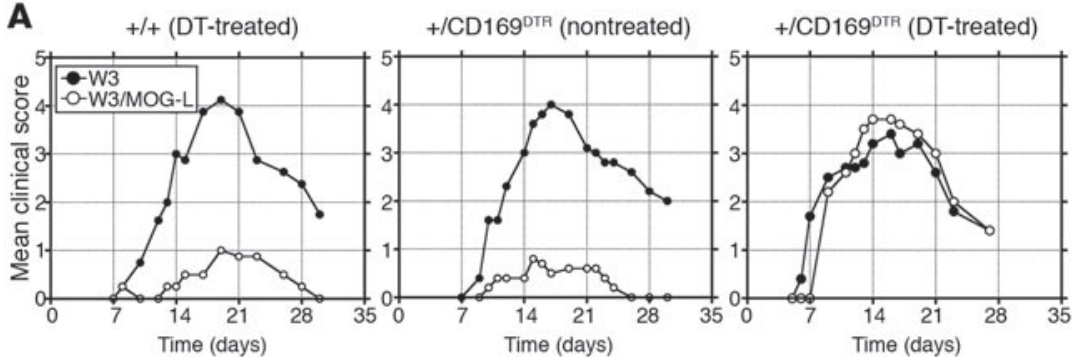

B

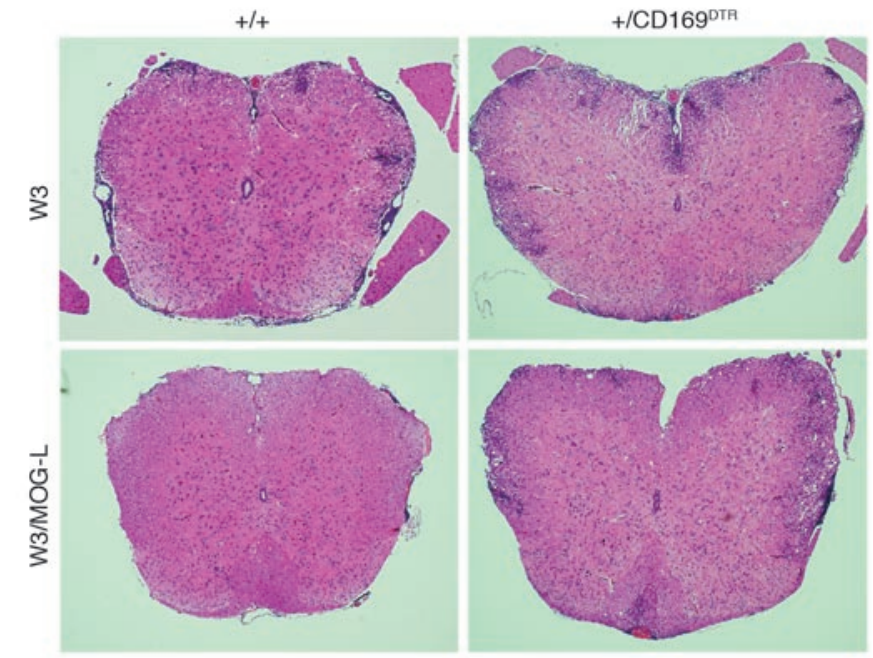

Figure 5

Failure of apoptotic cell-mediated tolerance induction after depletion of MZM. We intraperitoneally injected $10 \mu \mathrm{g} / \mathrm{kg}$ of DT into wild-type (+/+) or CD169-DTR (+/CD169DTR) mice 8 days prior to immunization. We intravenously injected $2 \times 10^{7}$ apoptotic W3 or W3/ MOG-L cells into mice 4 days prior to immunization with $\mathrm{MOG}_{35-55}$ in CFA on day 0 . (A) Disease severity of each mouse was scored, and the mean clinical scores at the indicated times were plotted. (B) Spinal cords were obtained 18 days after immunization, fixed with paraformaldehyde, and embedded in paraffin. Paraffin sections were stained with H\&E. Results are representative of 3 independent experiments. Original magnification, $\times 40$.

but instead found that injection of DT induced the deletion of not only MMM (CD169+ cells) but also of MZM (ER-TR9+ cells) (Figure 4C). To better define expression of CD169 mRNA, we prepared total RNA from both MMM and MZM using a microlaser dissecting system and performed RT-PCR with CD169-specific primers. The expression of CD169 mRNA was detected in both types of cells although the expression level in the MZM was lower than that in the MMM (data not shown), explaining why both cell types were depleted by DT administration. In contrast, $\mathrm{CD} 11 \mathrm{~b}^{+}$red pulp macrophages, TBM, T cells, B cells, and DCs were unaffected by DT administration (Figure 4C, Supplemental Figure 1, and data not shown. Supplemental material available online with this article; doi:10.1172/JCI31990DS1). We also examined the time course of macrophage depletion in CD169-DTR mice. When $10 \mu \mathrm{g} / \mathrm{kg}$ of DT was injected into the transgenic mice, both types of macrophages in the $\mathrm{MZ}$ were completely depleted within 2 days, and the depletion lasted until 7 days after DT injection (Figure 4D). We also analyzed other macrophages in the CD169-DTR mice treated with $10 \mu \mathrm{g} / \mathrm{kg}$ of DT. CD169-positive macrophages in LNs were also deleted in the mice by DT administration. Although the number of Kupffer cells in the liver was slightly reduced, no obvious changes were observed in macrophages in several organs 4 days after DT treatment.

Failure of tolerance induction in CD169-DTR mice. DT $(10 \mu \mathrm{g} / \mathrm{kg})$ was intraperitoneally administrated into the CD169-DTR mice 8 days prior to immunization. Four days later, apoptotic W3/ MOG-L cells were intravenously injected, and then the mice were immunized with $\mathrm{MOG}_{35-55}$ in CFA on day 0. Injection of apoptotic W3/MOG-L cells did not suppress EAE in the CD169-DTR mice treated with DT while injection of these cells inhibited EAE progression both in DTtreated wild-type littermates and in CD169DTR mice without DT treatment (Figure 5A). To independently confirm the severity of the EAE in the different mice, we histologically analyzed spinal cords of these mice. As shown in Figure $5 \mathrm{~B}$, a large number of lymphocytes infiltrated into spinal cords of wild-type littermates while the cellular infiltrate was nearly undetectable in wild-type littermates treated with apoptotic W3/MOG-L cells. However, a massive infiltration of lymphocytes was observed in DT-treated CD169-DTR mice even when the mice were pretreated with apoptotic W3/MOG-L cells. These results clearly indicate that macrophages in the $\mathrm{MZ}$ were indispensable for the induction of tolerance to cell-associated antigens.

Delayed clearance of injected apoptotic cells in CD169-DTR mice. To elucidate the mechanisms for the failure of tolerance induction in the DT-treated CD169-DTR mice, we determined how injected apoptotic cells were cleared in the spleen. Fluorescent-labeled apoptotic cells were injected intravenously, and the spleens were analyzed by immunohistochemistry. As shown in Figure 6, injected cell corpses accumulated in the $\mathrm{MZ}$ as early as 5 minutes after injection in wild-type mice. The corpses were located along with mucosal addressin cell adhesion molecule-1-positive (MAdCAM-1-positive) sinus lining cells and in both layers of macrophages in the $\mathrm{MZ}$ (ER-TR9 ${ }^{+}$and $\mathrm{CD} 169^{+}$cells). The fluorescence rapidly disappeared, and only a small number of corpses could be observed 2 hours after injection. In DT-treated CD169-DTR mice, injected apoptotic cells were also found along the sinus lining cells 5 minutes after injection even in the absence of both types of macrophages in the MZ. However, there was a dramatic delay in corpse clearance, and a significant number of fluorescent-labeled corpses still remained in the MZ even 2 hours after injection. These results indicate that macrophages in the $\mathrm{MZ}$ played a critical role in the clearance of intravenously injected apoptotic corpses.

Aberrant phagocytosis of apoptotic cells by $C D 11 b^{+} D C$ s in CD169-DTR mice. Previous studies have demonstrated that the $\mathrm{CD} 8 \alpha^{+} \mathrm{DC}$ subset plays a critical role in the uptake of apoptotic cells and presentation of cell-associated antigens $(12,22,23)$. Therefore, we examined the phagocytosis of injected apoptotic cells by DCs in 
A
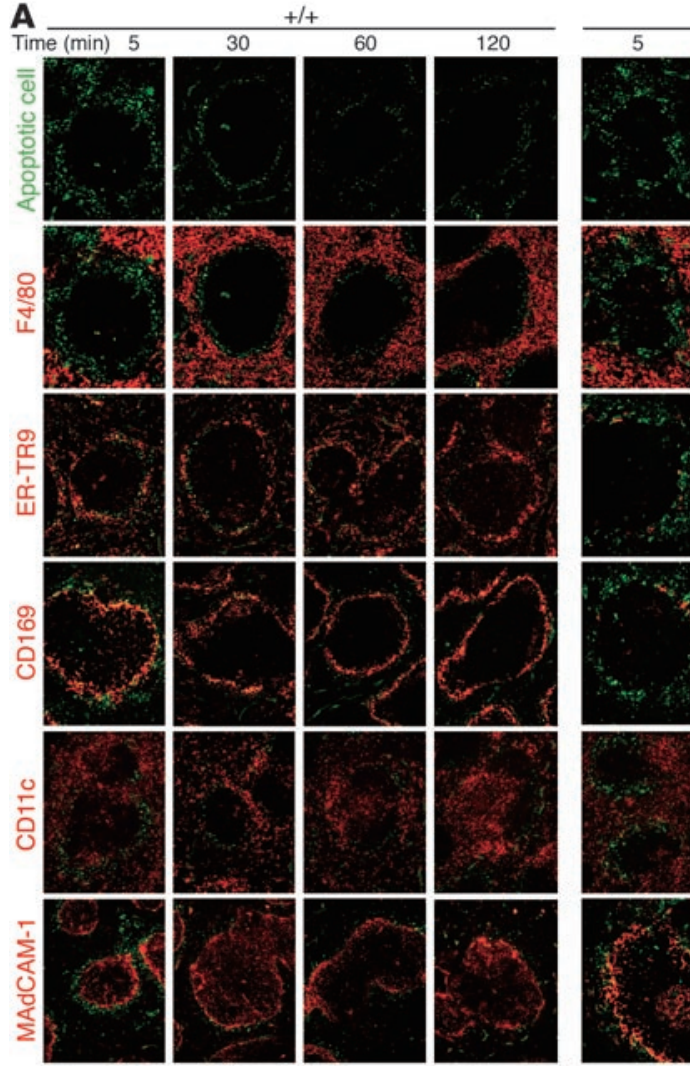

$\mathbf{B}$

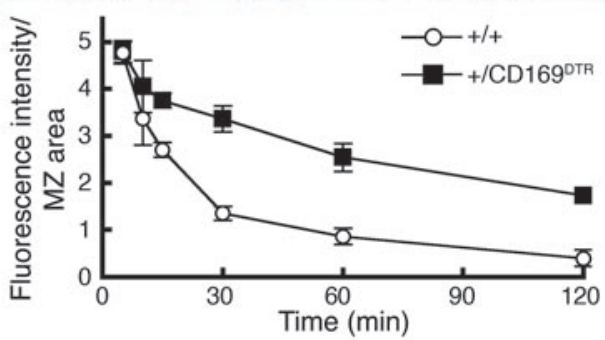

Figure 6

Delay of apoptotic cell clearance in DT-treated CD169-DTR mice. We intraperitoneally injected $10 \mu \mathrm{g} / \mathrm{kg}$ of DT into wild-type (+/+) or CD169-DTR (+/CD169DTR) mice. Four days after DT treatment, CMFDA-labeled (green) apoptotic W3/MOG-L cells $\left(2 \times 10^{7}\right.$ cells) were intravenously injected, and the spleen was obtained at the indicated times after injection. (A) Cryosections of the spleens were stained with F4/80 (red pulp macrophage), ER-TR9 (MZM), CD169 (MMM), CD11c (DC), or MAdCAM-1 (marginal sinus-lining cell) (red). (B) Fluorescence intensity in MZ on the slices was quantified at the indicated time points, and mean values are shown with SEM. These results are representative of 2 independent experiments. Original magnification, $\times 200$.

DT-treated CD169-DTR mice. We first analyzed the effects of DT administration on the population and distribution of splenic DCs. $\mathrm{CD} 11 \mathrm{c}^{+} \mathrm{DCs}$ were mainly localized in the $\mathrm{MZ}$ and $\mathrm{T}$ cell area of white pulp in wild-type mice (Figure 7A). When DT was injected into CD169-DTR mice, the CD11 $\mathrm{c}^{+}$DCs were still observed in the same areas, indicating that the distribution of DCs was unaffected in DT-treated CD169-DTR mice. We also examined the DC populations of DT-treated CD169-DTR mice by flow cytometry analysis. As shown in Figure 7B, neither the total population of CD $11 c^{+}$DCs nor the DC subsets (CD8 $\alpha^{+} \mathrm{CD} 11 \mathrm{~b}^{-}$lymphoid DCs and $C D 8 \alpha^{-} \mathrm{CD} 11 \mathrm{~b}^{+}$myeloid DCs) were affected by DT treatment in either wild-type or CD169-DTR mice.
Accordingly, we analyzed the phagocytosis of injected apoptotic cells by DCs in CD169DTR mice. Wild-type and CD169-DTR mice were treated with DT, and 4 days after treatment, fluorescence-labeled apoptotic cells were injected into mice. As shown in Figure 8, $A$ and $B$, apoptotic cells were mainly engulfed by $C D 8 \alpha^{+}$DCs, and only a small population of $\mathrm{CD} 11 \mathrm{~b}^{+} \mathrm{DCs}$ engulfed dead cell corpses in wildtype mice. On the other hand, the number of DCs engulfing apoptotic cells greatly increased in DT-treated CD169-DTR mice. In these mice, injected cell corpses were mainly phagocytosed by $\mathrm{CD} 11 \mathrm{~b}^{+}$myeloid DCs although the number of CD8 $\alpha^{+}$DCs engulfing dead cells was not different from that in wild-type mice. These results indicated that macrophages in the $\mathrm{MZ}$ regulated selective engulfment of apoptotic cells by CD $8 \alpha^{+}$DCs and that their functional failure caused the aberrant phagocytosis of apoptotic cells by CD $11 b^{+}$DCs.

We next examined the antigen presentation activity of DCs phagocytosing injected apoptotic cells in wild-type mice and DT-treated CD169-DTR mice. For this purpose, we established transformants expressing OVA fragments in the same manner as W3/MOG cells. Three hours after intravenous injection of W3/OVA cells into mice, we prepared CD $8 \alpha^{+}$and CD $11 b^{+}$ DCs from spleens and cultured these cells with OT-II cells from OVA-specific TCR transgenic mice. As shown in Figure 8C, the antigen presentation activity of CD $8 \alpha^{+}$DCs was stronger than that of CD $11 b^{+}$DCs in wild-type mice. On the other hand, the antigen presentation activity of $\mathrm{CD}_{11} \mathrm{~b}^{+} \mathrm{DCs}$ was stronger than that of CD $8 \alpha^{+}$ DCs in DT-treated CD169-DTR mice. These results are consistent with the observation that CD $11 b^{+}$DCs aberrantly phagocytose injected apoptotic cells in DT-treated CD169-DTR mice (Figure 8B) and indicate that both $\mathrm{CD} 8 \alpha^{+}$and

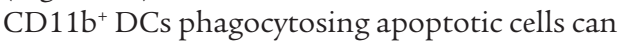
present cell-associated antigens to $\mathrm{T}$ cells.

\section{Discussion}

To maintain self tolerance, autoreactive T cells are initially eliminated in the thymus $(24,25)$.

To complement central tolerance, autoreactive $\mathrm{T}$ cells that escape central tolerance must be deleted in peripheral organs. Peripheral tolerance to self antigens is thought to be maintained by APCs, including DCs localized in peripheral tissues (26, 27). Resident DCs constantly phagocytose apoptotic cells generated during normal tissue turnover and migrate to draining LNs where they induce deletion or anergy of CD 4 and CD8 T cells by presentation of cell-associated antigens obtained from cell corpses. In addition to the peripheral tolerance induced by residual DCs in LNs, phagocytes in the spleen can also present self antigens derived from circulating dying cells to induce self tolerance. Here, we show that intravenous injection of apoptotic cells expressing a MOG fragment induced MOG-specific T cell tolerance and prevented 
A
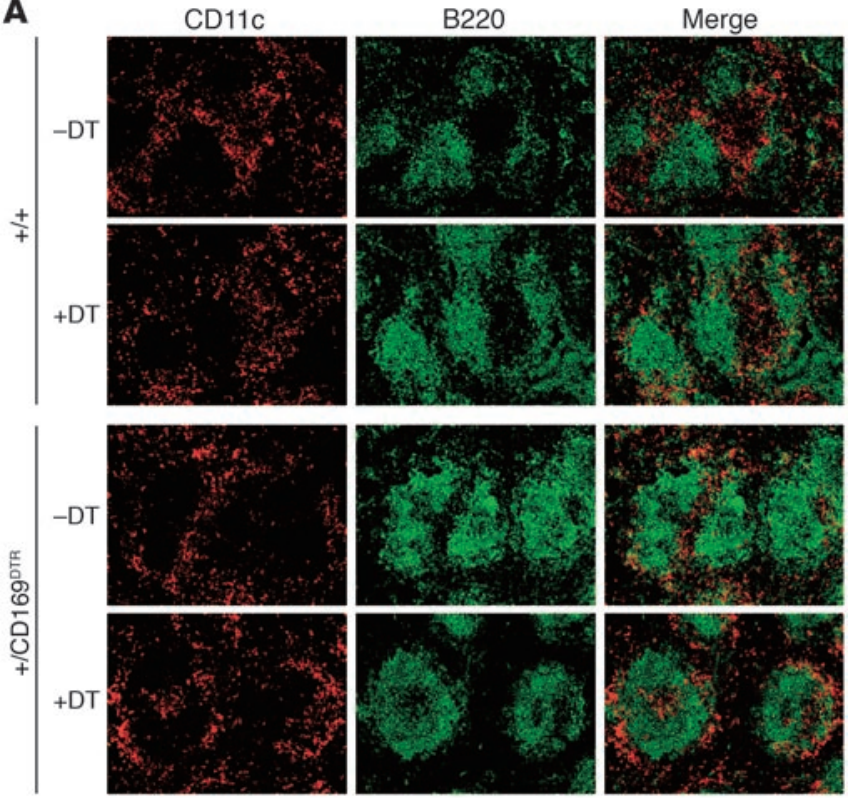

B

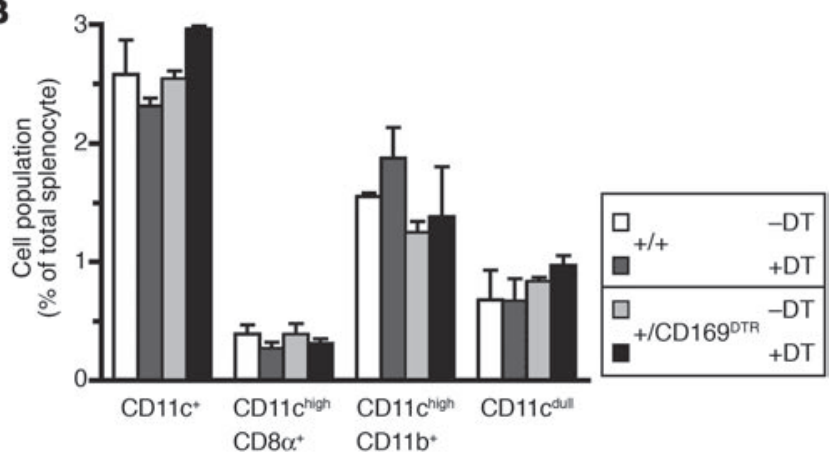

Figure 7

DCs were not affected by DT treatment in CD169-DTR mice. Wild-type $(+/+)$ or CD169-DTR (+/CD169DTR) mice were injected with (+DT) or without (-DT) $10 \mu \mathrm{g} / \mathrm{kg}$ of DT. Spleens were obtained 4 days after DT treatment. (A) Cryosections of the spleen were stained with CD11C (DCs) and B220 (B cells). Original magnification, $\times 200$. (B) Single-cell suspensions of splenocytes were stained with CD11c alone (total DCs) or in combination with CD8 $\alpha$ (lymphoid DCs) or CD11b (myeloid DCs) and analyzed by flow cytometry.

the development of EAE. The tolerance induction can also be achieved through injection of a large amount of soluble antigens. Compared with the amount of soluble antigens, smaller amounts of antigens were required for tolerance induction when they were associated with dead cell corpses. This efficiency may result from effective endocytosis of antigens in association with phagocytosis of apoptotic cell corpses. In addition, phagocytosis of apoptotic cells has immunoregulatory effects, including secretion of immunosuppressive cytokines such as TGF- $\beta$ and IL-10 $(28,29)$, which may promote immune tolerance to cell-associated antigens. Thus, delivery of antigens via injection of dying cells may be a valuable method to regulate immunological responses.

Because of its unique position at the interface between the lymphoid compartment of the spleen and the scavenging red pulp compartment, the $\mathrm{MZ}$ is well equipped to constantly screen the blood for foreign particles and organisms as well as aberrant molecular debris and dying cells $(30,31)$. The MZ contains 2 types of macrophages, MZM and MMM. MMM are localized at the inner border of the MZ close to the white pulp whereas MZM can be found at the outer rim, near the red pulp. MZM have potent phagocytic activity with the expression of several pattern-recognition receptors, suggesting that these cells are responsible for the recognition and clearance of invading microorganisms. One such receptor, the C-type lectin SIGN-R1, is expressed in mouse MZM and has a strong binding activity for the capsular polysaccharide of Streptococcus pneumoniae (32-34). When fluorescence-labeled $S$. pneumoniae were injected into wild-type mice, the labeled bacteria were exclusively localized in the $\mathrm{MZ}$ of spleen. However, in SIGN-R1-deficient mice, which showed increased susceptibility to $S$. pneumoniae infection, the injected bacteria were no longer restricted to the $\mathrm{MZ}$ and instead were disseminated throughout the red pulp (35). These results indicate that SIGN-R-expressing MZM are responsible for trapping and clearing circulating bloodborne bacteria. In the present study, the injected apoptotic cells rapidly accumulated into the $\mathrm{MZ}$ in wild-type mice. However, in contrast to blood-borne bacteria, this accumulation of injected corpses was still observed when macrophages in the $\mathrm{MZ}$ were transiently deleted. Even in the absence of functional macrophages in the MZ, apoptotic cell corpses did not disseminate into the red pulp, suggesting that there is a macrophage-independent mechanism for accumulation of circulating apoptotic cell corpses in the MZ. According to the 2-step model for apoptotic cell phagocytosis, apoptotic cells are first tethered to phagocytes through the interaction between putative ligands and receptors, then engulfed through recognition of PS exposed on the apoptotic cell surface (36). In this regard, tethering molecules for apoptotic cells may be expressed on other cells, such as the sinus lining cells in the $\mathrm{MZ}$, and tethered corpses may be recognized and phagocytosed by MMM, MZM, or both. Since both MMM and MZM were transiently deleted by DT administration in the CD169-DTR mice, we could not distinguish which cell type was responsible for apoptotic cell clearance in the MZ. Therefore, it will be of considerable interest to perform further analysis in mice with selective depletion of MMM or MZM.

In this study, transient deletion of macrophages in the $\mathrm{MZ}$ resulted in failure of induction of tolerance to apoptotic cell-associated antigens. This result strongly suggests that these cells play a critical role in induction of tolerance to cell-associated antigens. However, the underlying mechanisms responsible for the impairment of tolerance induction in the absence of these macrophages remain elusive. One possibility is that tolerance induction is blocked by the effects of endogenous adjuvants released from dead corpses. As deletion of macrophages in the MZ caused delayed clearance of dying cells, dying cells may undergo secondary necrosis in the MZ, and potentially immunogenic materials such as uric acid and heat shock proteins may be released to activate immunity $(37,38)$. Liu et al. reported that the tolerizing effects of DCs with cell-associated antigens could be overcome when a DC maturation signal, such as ligation of CD40, was simultaneously provided (12), suggesting that the presence of endogenous immunostimulatory molecules could interfere with induction of tolerance to cell-associated antigens.

Alternatively, aberrant phagocytosis of injected dying cells by $\mathrm{CD}_{11} \mathrm{~b}^{+}$myeloid DCs may interfere with induction of tolerance to cell-associated antigens in the DT-treated CD169-DTR mice. $\mathrm{CD} 8 \alpha^{+}$and $\mathrm{CD} 11 \mathrm{~b}^{+}\left(\mathrm{CD} 8 \alpha^{-}\right) \mathrm{DCs}$ are located in different regions of the spleen. Although the small population of CD8 $\alpha^{+}$DCs is 
A
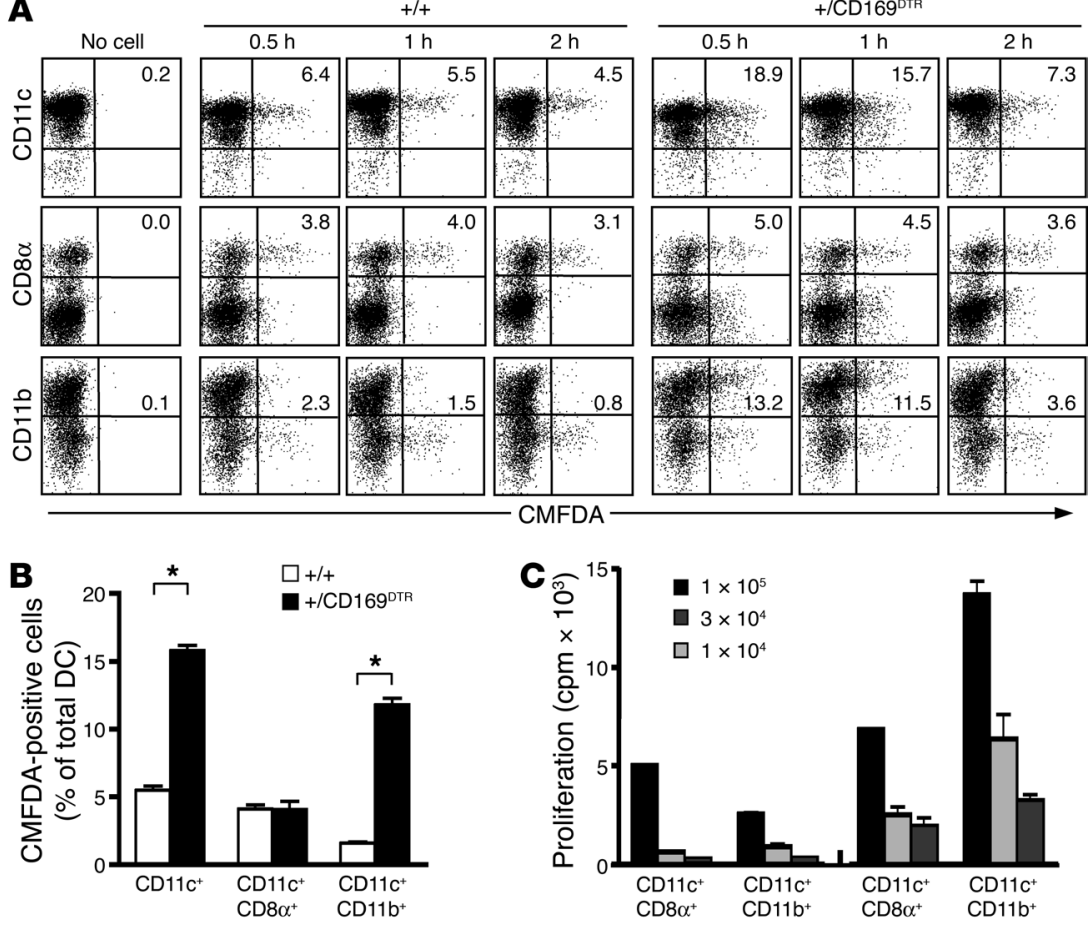

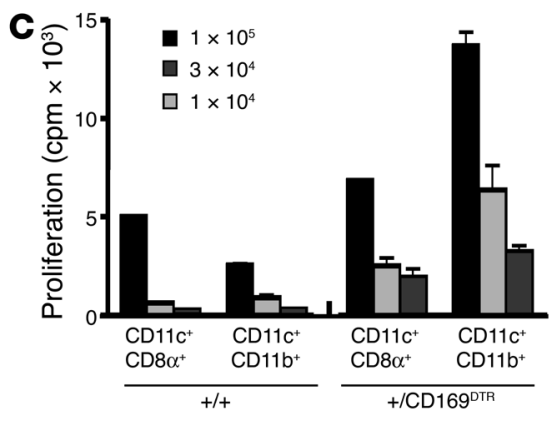

Figure 8

Aberrant engulfment of apoptotic cells by CD11b+ DCs in MZM-depleted mice. We intraperitoneally injected $10 \mu \mathrm{g} / \mathrm{kg}$ of DT into wild-type (+/+) or CD169-DTR (+/CD169DTR) mice. Four days after DT treatment, CMFDA-labeled apoptotic W3/MOG-L cells $\left(2 \times 10^{7}\right.$ cells) were intravenously injected, and the spleens were obtained at the indicated times after injection. Splenic DCs were enriched by cell sorting with anti-CD11c microbeads and stained with CD11c-PE, CD8 $\alpha-P E$, or CD11b-PE. PE and CMFDA double-positive cells were considered to have engulfed the injected apoptotic cells. (A) Kinetics of apoptotic cell engulfment by DC subsets. (B) Engulfment of apoptotic cells by DC subsets 1 hour after injection. These results are representative of 3 independent experiments, and mean values are shown with SD. ${ }^{\star} P<0.01$. (C) Antigen-presentation activity of $\mathrm{DC}$ subsets. We intravenously injected W3/OVA cells $\left(2 \times 10^{7}\right.$ cells $)$ into wild-type $(+/+)$ and DT-treated CD169-DTR (+/CD169DTR) mice, and spleen was obtained 3 hours after injection. CD11C ${ }^{+}$ DCs were further separated into CD $8 \alpha^{+}$DCs and CD11b+ DCs by the cell sorter. Indicated number of DC subsets was cocultured with OT-II cells $\left(2 \times 10^{5}\right.$ cells) for 60 hours. T cell proliferation was quantified based on $\left[{ }^{3} \mathrm{H}\right]$ thymidine uptake in the last 12 hours of the culture. These results are representative of 2 independent experiments, and mean values are shown with SD.

located in the red pulp and MZ, this DC subpopulation is predominantly found in the $\mathrm{T}$ cell-rich areas of the periarteriolar lymphatic sheaths (39). On the other hand, CD11 $\mathrm{b}^{+}$DCs are mainly located in the MZ. While both types of DCs have phagocytic activity for latex beads, under normal circumstances, the CD8 $\alpha^{+}$DCs preferentially engulf circulating dying cells and present cell-associated antigens to $\mathrm{T}$ cells (23). Our study has demonstrated that CD11 $\mathrm{b}^{+}$ DCs phagocytosed circulating apoptotic cells in the absence of macrophages in the MZ. This result suggests a role for $C D 11 b^{+}$DCs in the presentation of cell-associated antigens under pathological conditions. In vitro studies have demonstrated that CD $11 \mathrm{~b}^{+}$DCs induce stronger proliferative responses by $\mathrm{CD} 4$ and $\mathrm{CD} 8 \mathrm{~T}$ cells than $\mathrm{CD} 8 \alpha^{+} \mathrm{DCs}(40,41)$. In vivo studies have revealed that targeting antigens to different subsets of DCs induces distinct immune responses but delivery of antigens to both DC subsets without any maturation signals leads to $\mathrm{T}$ cell tolerance $(42,43)$. The other

group, on the other hand, has reported that targeting of antigens to $\mathrm{CD} 11 \mathrm{~b}^{+}$DCs was found to induce humoral immunity even in the absence of adjuvants (44). It should be informative to analyze the roles of CD $11 \mathrm{~b}^{+} \mathrm{DCs}$ in induction of tolerance to cell-associated antigens, particularly under pathological conditions.

In summary, our study has demonstrated that macrophages in the $\mathrm{MZ}$ of spleen regulate not only the efficient clearance of circulating apoptotic cell corpses but also selective engulfment of dying cells by CD $8 \alpha^{+}$DCs and that this regulation plays an important role in induction of tolerance to cell-associated antigens, one of the principle mechanisms for the maintenance of self tolerance.

\section{Methods}

Mice. C57BL/6J mice were obtained from Charles River Laboratories. CD11c-DTR transgenic mice (C57BL/6J background) and OT-II mice were kindly provided by D. Littman (New York University School of Medicine, New York, New York, USA) and by W.R. Heath (The Walter and Eliza Hall Institute, Parkville, Victoria, Australia), respectively. All mice were housed under specific pathogen-free conditions in the RIKEN Research Center for Allergy and Immunology (RCAI) animal facility. All experiments using mice described herein were approved by the RCAI animal use committee and performed in accordance with the applicable guidelines and regulations.

Establishment of transformants. To obtain the rat MOG cDNA, total RNA was isolated from the temporal lobe of a 6-week-old female Wistar rat (CLEA Japan Inc.). Since we established anti-DTR monoclonal antibodies with very good sensitivity and selectivity to DTR expressed on the cell surface (45), we chose DTR as the fusion partner of the MOG fragment to establish a cell line expressing the membrane-bound MOG fragment. The expression plasmids to generate chimeric transmembrane proteins between human HB-EGF and rat MOG (HB-EGF-MOG) were constructed by recombinant PCR. Mouse W3 cells (mouse Fas-expressing WR19L cells, a T cell line from BALB/c mice) were transfected by electroporation with the HB-EGF-MOG plasmid. Transformants were selected by flow cytometry using an anti-human HB-EGF monoclonal antibody. HB-EGF-MOG proteins in the transformants were detected by Western blotting using an anti-HB-EGF polyclonal antibody (R\&D Systems). In order to confirm the expression of the full-length HB-EGF-MOG/ FLAG fusion protein, cell lysates were immunoprecipitated with anti-FLAG antibody (Sigma-Aldrich) followed by Western blotting with anti-HB-EGF antibody or anti-MOG antibody (R\&D Systems).

To establish the transformants expressing the HB-EGF-OVA chimeric protein, cDNA encoding $\mathrm{OVA}_{2-386}$ was fused with the extracellular and transmembrane regions of HB-EGF cDNA. The expression plasmids were introduced into mouse W3 cells as described above.

Administration of apoptotic cells. W3 cells or their transformants were treated for 4 hours with $1 \%$ culture supernatant of Fas ligand-expressing cells (46) in RPMI 1640/1\% syngeneic mouse serum. Cells were then washed twice with PBS containing $1 \mathrm{mg} / \mathrm{ml}$ mouse serum albumin (Sigma-Aldrich) in 
order to avoid any contamination with bovine serum. To confirm that the cells had undergone apoptosis, cells were stained with annexin V-PE (BD Biosciences) and analyzed by flow cytometry. Indicated numbers of apoptotic cells in $200 \mu \mathrm{l}$ of PBS $/ 2.5 \%$ syngeneic mouse serum were intravenously injected into mice through the tail vein or subcutaneously injected into the tail base. In some experiments, the recombinant MFG-E8 mutant proteins D89E and E1E2PT, prepared as described previously (17), were added to apoptotic cells prior to injection.

Induction of EAE. Female mice of 8 to 12 weeks of age were immunized by subcutaneous administration at 2 sites in the tail base of each mouse with $100 \mu \mathrm{g}$ of $\mathrm{MOG}_{35-55}$ peptide (MEVGWYRSPFSRVVHLYRNGK: NeoMPS) in $100 \mu \mathrm{l}$ PBS emulsified in $100 \mu \mathrm{l} \mathrm{CFA} \mathrm{(Difco;} \mathrm{BD} \mathrm{Diagnostics)} \mathrm{on} \mathrm{day} \mathrm{0,}$ supplemented with intravenous injection of $200 \mathrm{ng}$ of pertussis toxin (Seikagaku Corp.) in $200 \mu \mathrm{l}$ PBS. Mice received an additional intraperitoneal injection of $200 \mathrm{ng}$ pertussis toxin on day 2. Mice were observed for the presence of neurological signs of EAE and scored as follows: 0 , no symptoms; 1 , weak tail; 2 , complete loss of tail tone; 3 , ataxic gate; 4 , hind limb paralysis; 5 , hind and forelimb paralysis; 6 , death. Data are presented as mean clinical scores for each group.

$T$ cell proliferation assay. Mice preinjected with or without apoptotic cells were immunized in the bilateral foot pads with $100 \mu \mathrm{g}_{\text {of }} \mathrm{MOG}_{35-55}$ or $\mathrm{KLH}$ (Sigma-Aldrich) in CFA. Five days after immunization, popliteal and inguinal LNs were isolated. $\mathrm{LN}$ cells $\left(5 \times 10^{5}\right.$ cells $)$ were restimulated with $\mathrm{MOG}_{35-55}$ or $\mathrm{KLH}$ for 70 hours in vitro. Proliferation was quantified based on $\left[{ }^{3} \mathrm{H}\right]$ thymidine $(0.5 \mu \mathrm{Ci} /$ well $)$ uptake in the last 20 hours of the culture. Generation of CD169-DTR mice. A genomic clone containing the CD169 gene was obtained from a BAC library derived from R1 ES cell DNA (the F1 offspring of 129X1/SvJ and 129S1/Sv mice) (47). To generate the targeting vector, the region from -1.6 to $+8.2 \mathrm{kbp}$ relative to the transcriptional start site of the CD169 gene was subcloned into the pBluescript II SK (+) vector (Stratagene). Then a 28-bp fragment including the endogenous ATG start site in exon 1 was replaced with human DTR (HB-EGF) CDNA with a polyA tail by a recombinant PCR technique. To allow selection for homologous recombinants, a loxP-flanked Neo cassette was cloned downstream of the human DTR gene. The thymidine kinase gene was inserted downstream of the $3^{\prime}$ arm to select against random integrants. To generate CD169-DTR mice, R1 ES cells were transfected with the linearized targeting vector by electroporation. G-418- and ganciclovir-resistant clones were screened for homologous recombination by Southern blot analysis with a 680-bp DIG labeled DNA probe located outside the targeting vector using a DIG DNA Labeling Kit and Detection Kit (Roche Diagnostics). Germline chimeric mice were generated by aggregation methods. Chimeric mice with a high ES cell contribution were crossed with C57BL/6J mice to produce heterozygous CD169-DTR mice. The heterozygous CD169-DTR mice were backcrossed to C57BL/6J mice for more than 4 generations, and the wild-type $(+/+)$ and heterozygous CD169-DTR (+/CD169 DTR) littermates were used for analysis.

Immunohistochemistry. Cryosections $(4 \mu \mathrm{m})$ of the spleen were fixed in cold acetone and blocked with $1.5 \%$ normal goat serum and $2 \%$ Block Ace (Snow Bland Milk Products). Slides were incubated with anti-CD169 (3d6.112; AbD Serotec), MAdCAM-1 (MECA-367; eBioscience) antibodies, biotinylated anti-CD11b (M1/70; BD Biosciences), or biotinylated ER-TR9 (BMA Biomedicals) antibodies. The sections were then incubated with Cy3-anti-rat IgG (Jackson ImmunoResearch Laboratories Inc.) antibody for CD169 and MAdCAM-1 or Cy3-streptavidin (Jackson ImmunoResearch Laboratories Inc.) for CD11b and ER-TR9. Stained sections were mounted with FluorSave (Calbiochem; EMD Biosciences) and observed by fluorescence microscopy (Olympus IX-71). For double staining, sections were stained with biotinylated anti-CD11c antibody (HL3; BD Biosciences) followed by Cy3-streptavidin and Alexa Fluor 488-anti-B220 antibody (RA3-6B2; BD Biosciences).
Cytokine ELISA. Mice preinjected with apoptotic cells were immunized with $\mathrm{MOG}_{35-55}$ in CFA. The spleen was removed on day 10 , and single cell suspension of splenocytes $\left(5 \times 10^{5}\right.$ cells $)$ was restimulated with MOG $_{35-55}$ peptide for 72 hours in vitro. Concentration of IFN- $\gamma$ and IL-17 in the culture medium was measured by ELISA (BD Biosciences; R\&D Systems) according to the manufacturers' protocols.

In vivo distribution of injected apoptotic cells. W3/MOG-L cells were labeled with $5 \mu \mathrm{M}$ of 5-chloromethylfluorescein diacetate (CMFDA) (CellTracker Green; Invitrogen). Apoptosis was induced in the labeled cells, and the cells were washed as described above. We intravenously injected $2 \times 10^{7}$ CMFDAlabeled apoptotic W3/MOG-L cells in $200 \mu \mathrm{lBS} / 2.5 \%$ mouse serum into DT-treated wild-type and CD169-DTR mice. Spleens were obtained at various time points after the injection. The distribution of injected apoptotic cells was analyzed by immunohistochemistry and by flow cytometry. For immunohistochemical assessments, $4-\mu \mathrm{m}$ cryosections were stained with anti-F4/80 (Invitrogen), ER-TR9, CD169, or MAdCAM-1 antibodies. The fluorescence intensity in $\mathrm{MZ}$ on the slices was quantified by using MetaMorph software (version 7.1; Molecular Devices). For flow cytometric analysis, spleens were flushed with $100 \mathrm{U} / \mathrm{ml}$ collagenase D (Roche Diagnostics), teased apart with fine forceps, and digested with $400 \mathrm{U} / \mathrm{ml}$ collagenase $\mathrm{D}$ for 45 minutes at $37^{\circ} \mathrm{C}$. After digestion, splenic DCs were enriched by MACS sorting with antiCD11c microbeads (Miltenyi Biotec). CD11 $\mathrm{c}^{+}$DCs were then stained with CD11c-PE (total DCs), CD8 $\alpha$-PE (lymphoid DCs), or CD11b-PE (myeloid DCs) and analyzed by flow cytometry. Cells positive for both PE and CMFDA were considered to have engulfed injected apoptotic cells.

Antigen presentation by DC subsets. W3/OVA cells $\left(2 \times 10^{7}\right.$ cells) were intravenously injected into wild-type and DT-treated CD169-DTR mice, and the spleen was obtained 3 hours after injection. DCs were enriched by cell sorting with anti-CD $11 \mathrm{c}$ microbeads. CD $11 \mathrm{c}^{+} \mathrm{DCs}$ were further separated into CD8 $\alpha^{+}$DCs and CD $11 b^{+}$DCs by the cell sorter. OVA-specific, MHC class IIrestricted, OT-II TCR transgenic T cells were purified from spleen and LNs of OT-II mice by anti-CD 4 microbeads. The graded dose of DCs was cocultured with OT-II cells $\left(2 \times 10^{5}\right.$ cells) for 60 hours. T cell proliferation was quantified based on $\left[{ }^{3} \mathrm{H}\right]$ thymidine uptake in the last 12 hours of the culture.

Statistics. All the statistical analyses were performed by using an unpaired 2 -tailed Student's $t$ test. A $P$ value of less than 0.05 was considered significant.

\section{Acknowledgments}

We thank D. Littman for CD11c-DTR mice; W.R. Heath for OT-II mice; S. Nagata for pEF-BOS-EX vector, W3 cells, recombinant MFG-E8 proteins, and soluble Fas ligand; K. Kohno for human HB-EGF cDNA; H. Fujimoto and Y. Hachiman for cell sorting; and H. Matsuda for secretarial assistance. We also thank P.D. Burrows, T. Kurosaki, and T. Takemori for critical comments. This work was supported in a part by a grant-in-aid from the Ministry of Education, Culture, Sports, Science and Technology (MEXT) in Japan, by the Uehara Memorial Foundation, and by the Mochida Memorial Foundation for Medical and Pharmaceutical Research.

Received for publication March 1, 2007, and accepted in revised form May 9, 2007.

Address correspondence to: Masato Tanaka, Laboratory for Innate Cellular Immunity, RIKEN Research Center for Allergy and Immunology, 1-7-22, Suehiro, Tsurumi, Yokohama, Kanagawa 2300045, Japan. Phone: 81-45-503-9686; Fax: 81-45-503-9685; E-mail: mtanaka@rcai.riken.jp.

Yasunobu Miyake and Kenichi Asano contributed equally to this work. 
1. Vaux, D.L., and Korsmeyer, S.J. 1999. Cell death in development. Cell. 96:245-254.

2. Nagata, S. 1997. Apoptosis by death factor. Cell. 88:355-365.

3. Henson, P.M., Bratton, D.L., and Fadok, V.A. 2001 Apoptotic cell removal. Curr. Biol. 11:R795-R805.

4. Hengartner, M.O. 2001. Apoptosis: corralling the corpses. Cell. 104:325-328.

5. Savill, J., and Fadok, V. 2000. Corpse clearance defines the meaning of cell death. Nature. 407:784-788.

6. Maderna, P., and Godson, C. 2003. Phagocytosis of apoptotic cells and the resolution of inflammation. Biochim. Biophys. Acta. 1639:141-151.

7. Lauber, K., Blumenthal, S.G., Waibel, M., and Wesselborg, S. 2004. Clearance of apoptotic cells: getting rid of the corpses. Mol. Cell. 14:277-287.

8. Scott, R.S., et al. 2001. Phagocytosis and clearance of apoptotic cells is mediated by MER. Nature. 411:207-211

9. Hanayama, R., et al. 2002. Identification of a factor that links apoptotic cells to phagocytes. Nature. 417:182-187.

10. Hanayama, R., et al. 2004. Autoimmune disease and impaired uptake of apoptotic cells in MFGE8-deficient mice. Science. 304:1147-1150.

11. Cohen, P.L., et al. 2002. Delayed apoptotic cell clearance and lupus-like autoimmunity in mice lacking the c-mer membrane tyrosine kinase. J. Exp. Med. 196: $135-140$

12. Liu, K., et al. 2002. Immune tolerance after delivery of dying cells to dendritic cells in situ. J. Exp. Med. 196:1091-1097.

13. Sun, E., et al. 2004. Allograft tolerance induced by donor apoptotic lymphocytes requires phagocytosis in the recipient. Cell Death Differ. 11:1258-1264.

14. Saito, M., et al. 2001. Diphtheria toxin receptormediated conditional and targeted cell ablation in transgenic mice. Nat. Biotechnol. 19:746-750.

15. Gammon, G., and Sercarz, E. 1989. How some T cells escape tolerance induction. Nature. 342:183-185.

16. Kaufman, D.L., et al. 1993. Spontaneous loss of T-cell tolerance to glutamic acid decarboxylase in murine insulin-dependent diabetes. Nature. 366:69-72.

17. Asano, K., et al. 2004. Masking of phosphatidylserine inhibits apoptotic cell engulfment and induces autoantibody production in mice. J. Exp. Med. 200:459-467.

18. Jung, S., et al. 2002. In vivo depletion of CD11c(+) dendritic cells abrogates priming of CD8 $(+) \mathrm{T}$ cells by exogenous cell-associated antigens. Immunity. 17:211-220
19. Probst, H.C., et al. 2005. Histological analysis of CD11c-DTR/GFP mice after in vivo depletion of dendritic cells. Clin. Exp. Immunol. 141:398-404

20. Crocker, P.R., and Gordon, S. 1989. Mouse macrophage hemagglutinin (sheep erythrocyte receptor) with specificity for sialylated glycoconjugates characterized by a monoclonal antibody. J. Exp. Med. 169:1333-1346.

21. Crocker, P.R., et al. 1994. Sialoadhesin, a macrophage sialic acid binding receptor for haemopoietic cells with 17 immunoglobulin-like domains. EMBO J. 13:4490-4503.

22. Ferguson, T.A., et al. 2002. Uptake of apoptotic antigen-coupled cells by lymphoid dendritic cells and cross-priming of CD8(+) $\mathrm{T}$ cells produce active immune unresponsiveness. J. Immunol. 168:5589-5595.

23. Iyoda, T., et al. 2002. The CD8+ dendritic cell subset selectively endocytoses dying cells in culture and in vivo. J. Exp. Med. 195:1289-1302.

24. Goldrath, A.W., and Bevan, M.J. 1999. Selecting and maintaining a diverse T-cell repertoire. Nature. 402:255-262.

25. Kyewski, B., and Klein, L. 2006. A central role for central tolerance. Annu. Rev. Immunol. 24:571-606.

26. Heath, W.R., et al. 2004. Cross-presentation, dendritic cell subsets, and the generation of immunity to cellular antigens. Immunol. Rev. 199:9-26.

27. Steinman, R.M., Hawiger, D., and Nussenzweig, M.C. 2003. Tolerogenic dendritic cells. Annu. Rev. Immunol. 21:685-711.

28. Fadok, V.A., et al. 1998. Macrophages that have ingested apoptotic cells in vitro inhibit proinflammatory cytokine production through autocrine/ paracrine mechanisms involving TGF-beta, PGE2, and PAF. J. Clin. Invest. 101:890-898.

29. Voll, R.E., et al. 1997. Immunosuppressive effects of apoptotic cells. Nature. 390:350-351.

30. Lopes-Carvalho, T., Foote, J., and Kearney, J.F. 2005 Marginal zone B cells in lymphocyte activation and regulation. Curr. Opin. Immunol. 17:244-250.

31. Mebius, R.E., and Kraal, G. 2005. Structure and function of the spleen. Nat. Rev. Immunol. 5:606-616.

32. Geijtenbeek, T.B., et al. 2002. Marginal zone macrophages express a murine homologue of DC-SIGN that captures blood-borne antigens in vivo. Blood. 100:2908-2916.

33. Kang, Y.S., et al. 2004. The C-type lectin SIGN-R1 mediates uptake of the capsular polysaccharide of Streptococcus pneumoniae in the marginal zone of mouse spleen. Proc. Natl. Acad. Sci. U.S. A. 101:215-220
34. Kang, Y.S., et al. 2003. SIGN-R1, a novel C-type lectin expressed by marginal zone macrophages in spleen, mediates uptake of the polysaccharide dextran. Int. Immunol. 15:177-186.

35. Lanoue, A., et al. 2004. SIGN-R1 contributes to protection against lethal pneumococcal infection in mice. J. Exp. Med. 200:1383-1393.

36. Hoffmann, P.R., et al. 2001. Phosphatidylserine (PS) induces PS receptor-mediated macropinocytosis and promotes clearance of apoptotic cells. J. Cell Biol. 155:649-659.

37. Millar, D.G., et al. 2003. Hsp70 promotes antigenpresenting cell function and converts T-cell tolerance to autoimmunity in vivo. Nat. Med. 9:1469-1476.

38. Shi, Y., Evans, J.E., and Rock, K.L. 2003. Molecular identification of a danger signal that alerts the immune system to dying cells. Nature. 425:516-521.

39. Neuenhahn, M., et al. 2006. CD8alphat dendritic cells are required for efficient entry of Listeria monocytogenes into the spleen. Immunity. 25:619-630.

40. Kronin, V., et al. 1996. A subclass of dendritic cells regulates the response of naive CD8 T cells by limiting their IL-2 production. J. Immunol. 157:3819-3827.

41. Suss, G., and Shortman, K. 1996. A subclass of dendritic cells kills CD4 T cells via Fas/Fas-ligandinduced apoptosis. J. Exp. Med. 183:1789-1796.

42. Bonifaz, L., et al. 2002. Efficient targeting of protein antigen to the dendritic cell receptor DEC-205 in the steady state leads to antigen presentation on major histocompatibility complex class I products and peripheral CD8+ T cell tolerance. J. Exp. Med. 196:1627-1638

43. Dudziak, D., et al. 2007. Differential antigen processing by dendritic cell subsets in vivo. Science. 315:107-111.

44. Corbett, A.J., et al. 2005. Antigen delivery via two molecules on the CD8- dendritic cell subset induces humoral immunity in the absence of conventional “danger.” Eur. J. Immunol. 35:2815-2825.

45. Miyake, Y., et al. 2007. Protective role of macrophages in noninflammatory lung injury caused by selective ablation of alveolar epithelial type II cells. J. Immunol. 178:5001-5009.

46. Shiraishi, T., et al. 2004. Increased cytotoxicity of soluble Fas ligand by fusing isoleucine zipper motif. Biochem. Biophys. Res. Commun. 322:197-202.

47. Nakayama, M., Iida, M., Koseki, H., and Ohara, O. 2006. A gene-targeting approach for functional characterization of KIAA genes encoding extremely large proteins. FASEB J. 20:1718-1720. 